\title{
3-D Object Positioning from Monocular Image Brightnesses
}

\author{
Tadayoshi SHIOYAMA, Hai Yuan WU, \\ Wen Biao JIANG and Susumu TERAUCHI \\ Department of Mechanical and System Engineering \\ Kyoto Institute of Technology \\ Matsugasaki, Sakyo-ku, Kyoto 606, JAPAN \\ FAX: 075-724-7300, Email: shioyama@ipc.kit.ac.jp
}

\begin{abstract}
This paper proposes an estimation scheme for threedimensional (3-D) object positioning from monocular image brightnesses without point correspondences while previous 3-D positioning methods need point correspondences. In the present scheme, at the first step, 3-D shapes of object are recovered from image brightnesses before and after rotation motion. At the second step, two vectors which are transformed according to rotation matrix under $3-D$ rotation, are constructed from central moments of the recovered 3-D shapes of object before and after motion, respectively. At the third step, rotation matrix is computed from these vectors by singular value decomposition. At the fourth step, from the rotation matrix the rotation axis and the rotation angle around the axis are estimated.
\end{abstract}

\section{Introduction}

Object positioning is an important area of research in computer vision. Estimation schemes for three-dimensional(3-D) object positioning from two image frames with a single camera have been developed in literatures [1]-[5]. However, in the previous estimation schemes it is assumed that image point (or line) correspondences are known. It is time-consuming and difficult to solve point correspondence problem for the case of many points. Therefore, it is desired to develope an estimation method for 3-D object positioning without point correspondences.

This paper proposes an estimation scheme for 3-D object positioning from two monocular image brightnesses. The present scheme has an advantageous property that it does not need point correspondences. In the present scheme, at the first step, 3-D shapes of object are recovered from image brightnesses before and after motion, respectively. At the second step, two vectors which are transformed according to rotation matrix under $3-D$ rotation, are constructed from central moments of the recovered 3-D shapes of object before and after motion, respectively. At the third step, rotation matrix is computed from these vectors by singular value decomposition(SVD). At the fourth step, from the rotation matrix the rotation axis and the rotation angle around the axis are estimated. The present scheme is evaluated quantitatively. 


\section{3-D Shape Recovery}

We assume orthographic image projection in observing objects and take the viewing direction to be parallel to the $z$-axis. Then, the shape of the object can be described by its height $z$ at coordinate $(x, y)$ in the image plane. The coordinate $(x, y, z)$ is called the camera coordinate. We denote by $\mathbf{n}_{i}$ a unit vector with the direction of light, and by $\mathbf{n}$ a unit vector normal to the surface of the object. The vector $\mathbf{n}$ is called the surface normal. The unit vectors $n$ and $n_{i}$ are described by points on the sphere with unit radius called Gaussian sphere. The stereographic projection is given as the projection of points on the Gaussian sphere through rays from the south pole onto a tangent plane (which is called the stereographic plane) at the north pole. The coordinates $(f, g)$ in the stereographic plane are given as follows:

$$
\begin{aligned}
& f=2 p\left[\sqrt{1+p^{2}+q^{2}}-1\right] /\left(p^{2}+q^{2}\right), \\
& g=2 q\left[\sqrt{1+p^{2}+q^{2}}-1\right] /\left(p^{2}+q^{2}\right),
\end{aligned}
$$

where

$$
p \equiv \partial z / \partial x, \quad q \equiv \partial z / \partial y \text {. }
$$

Unit vectors $n$ and $n_{i}$ are described in terms of $f$ and $g$ as follows:

$$
\begin{gathered}
\mathbf{n}=\left(-4 f,-4 g, 4-f^{2}-g^{2}\right) /\left(4+f^{2}+g^{2}\right), \\
\mathbf{n}_{i}=\left(-4 f_{i},-4 g_{i}, 4-f_{i}^{2}-g_{i}^{2}\right) /\left(4+f_{i}^{2}+g_{i}^{2}\right) .
\end{gathered}
$$

We assume that the viewing direction coincides with the north pole of the Gaussian sphere and that points on the northern hemisphere of the Gaussian sphere are considered. Therefore, the considered points $(f, g)$ and $\left(f_{i}, g_{i}\right)$ in the stereographic plane are constrained to the following regions:

$$
f^{2}+g^{2} \leq 4, \quad f_{i}^{2}+g_{i}^{2} \leq 4
$$

We denote by $F\left(f, g ; f_{1}, g_{i}\right)$ or $\mathrm{F}(\mathrm{f}, \mathrm{g})$ the reflectance map. Let $E_{i j}$ denote the observed brightness at a point with coordinate $(i, j)$ in the image plane, where $E_{i}$, is normalized so that the maximum observed brightness is equal to 1 . Then, it holds that

$$
F\left(f_{i j}, g_{i j} ; f_{i}, g_{i}\right)=E_{i j} \text {, }
$$

where $\left(f_{i j}, g_{i j}\right)$ represents the stereographic coordinate of the surface normal at the point with coordinate $(i, j)$ in the image. Denote by $\left(f_{i j}^{(k)}, g_{i j}^{(k)}\right)$ the estimated $\left(f_{i j}, g_{i j}\right)$ at $k$-th iteration and by $\left(f_{i j}^{*}, g_{i j}^{*}\right)$ the true $\left(f_{i y}, g_{i j}\right)$. We assume the following relation:

$$
\begin{aligned}
& f_{i \jmath}^{*}=f_{i \jmath}^{(k)}+\Delta f_{i j}^{(k)}, \\
& g_{i j}^{*}=g_{i \jmath}^{(k)}+\Delta g_{i j}^{(k)} .
\end{aligned}
$$

Here, $\Delta f_{i j}^{(k)}$ and $\Delta g_{i j}^{(k)}$ are the improvement at k-th iteration. We obtain $\Delta f_{i j}^{(k)}$ and $\Delta g_{i j}^{(k)}$ by Marquardt method with the constraint (7) as follows.

$$
\begin{aligned}
& \Delta f_{i j}^{(k)}=\frac{\lambda\left[E_{i j}-F\left(f_{i j}^{(k)}, g_{i j}^{(k)}\right)\right] F_{f}\left(f_{i j}^{(k)}, g_{i j}^{(k)}\right)}{1+\lambda\left[F_{f}\left(f_{i j}^{(k)}, g_{i j}^{(k)}\right)^{2}+F_{g}\left(f_{i j}^{(k)}, g_{i j}^{(k)}\right)^{2}\right]}, \\
& \Delta g_{i j}^{(k)}=\frac{\lambda\left[E_{i j}-F\left(f_{i j}^{(k)}, g_{i j}^{(k)}\right)\right] F_{g}\left(f_{i j}^{(k)}, g_{i j}^{(k)}\right)}{1+\lambda\left[F_{f}\left(f_{i j}^{(k)}, g_{i j}^{(k)}\right)^{2}+F_{g}\left(f_{i j}^{(k)}, g_{i j}^{(k)}\right)^{2}\right]},
\end{aligned}
$$


where

$$
F_{f} \equiv \frac{\partial F}{\partial f_{i j}}, \quad F_{g} \equiv \frac{\partial F}{\partial g_{i j}} .
$$

Hence, the iterative algorithm for inferring $\left(f_{i j}, g_{i j}\right)$ is given by

$$
\begin{aligned}
& f_{i j}^{(k+1)}=f_{i j}^{(k)}+\Delta f_{i j}^{(k)}, \\
& g_{i j}^{(k+1)}=g_{i j}^{(k)}+\Delta g_{i j}^{(k)},
\end{aligned}
$$

here $\Delta f_{i j}^{(k)}$ and $\Delta g_{i j}^{(k)}$ are given by (10) and (11).

The line of sight lies in the tangent plane at a point on the occluding boundary. Since we assume an orthographic image projection, the line of sight is perpendicular to the image plane. Hence, the tangent plane at a point on the occluding boundary is perpendicular to the image plane and is projected as a line in the image plane. This line is tangent to the silhouette of the occluding boundary in the image plane. Thus, a normal to the silhouette in the image plane is parallel to the surface normal at the corresponding point on the occluding boundary. That is, $\left(f_{i j}, g_{i j}\right)$ is known at the point $(i, j)$ corresponding to the occluding boundary. In the 3-D shape inference algorithm, at the initial iteration $(\mathrm{k}=0)$ only $\left(f_{i j}, g_{i j}\right)$ corresponding to the occluding boundary are known, and $\left(f_{i j}, g_{i j}\right)$ in the region other than the occluding boundary are set as $f_{i j}=g_{i j}=0$. The region where $f_{i j}=g_{i j}=0$ and $\left(f_{i j}, g_{i j}\right)$ is not yet improved, is called "unknown region". In the unknown region, for $k \neq 0$, we approximate $f_{i j}^{(k)}$ and $g_{i j}^{(k)}$ by the following steps (1) and (2).

(1) In the case where at least one of 8-neighbors of point $(i, j)$ belong to the known region,

$$
f_{i j}^{(k)}=a \bar{f}_{i j}^{(k)}+b \hat{f}_{i j}^{(k)}, \quad g_{i j}^{(k)}=a \bar{g}_{i j}^{(k)}+b \hat{g}_{i j}^{(k)},
$$

where

$$
\begin{aligned}
& \tilde{f}_{i j}=f_{i+1, j}+f_{i, j+1}+f_{i-1, j}+f_{i, j-1}, \\
& \bar{g}_{i j}=g_{i+1, j}+g_{i, j+1}+g_{i-1, j}+g_{i, j-1}, \\
& \hat{f}_{i j}=f_{i-1, j-1}+f_{i+1, j-1}+f_{i-1, j+1}+f_{i+1, j+1}, \\
& \hat{g}_{i j}=g_{i-1, j-1}+g_{i+1, j-1}+g_{i-1, j+1}+g_{i+1, j+1},
\end{aligned}
$$

$(a, b)=\left(1 / c_{1}, 0\right)$ when at least one of 4 -neighbors belong to the known region or $(a, b)=\left(0,1 / c_{2}\right)$ otherwise. $c_{1}$ (or $\left.c_{2}\right)$ denotes the number of 4 (or 8 -) neighbors belonging to the known region.

(2) In the case other than the case of (1),

$$
f_{i j}^{(k)}=g_{i j}^{(k)}=0 \text {. }
$$

In this case, we set as $\Delta f_{i j}^{(k)}=\Delta g_{i j}^{(k)}=0$.

As the iterative algorithm proceeds, the unknown region decreases. Hence, the steps (1) and (2) are only the initial transient process. After obtaining $(f, g)$ by the above algorithm, we can compute $(p, q)$ from equations (1) and (2), and we can obtain the height $\mathrm{z}$ by integrating $\mathrm{p}$ and $\mathrm{q}$. Thus we can recover $3-\mathrm{D}$ shape.

\section{Vectors Derived from Moments}

We define a moment of order $(\ell+m+n)$ as follows

$$
M_{\ell m n} \equiv \int_{-\infty}^{\infty} \int_{-\infty}^{\infty} \int_{-\infty}^{\infty} x^{\ell} y^{m} z^{n} \rho(x, y, z) d x d y d z
$$


where $\rho(x, y, z)$ denotes the density function of a 3-D object, and it is assumed that the origin of the coordinate system coincides with the centroid of the density function.

We consider a unit vector $\vec{u} \equiv\left(u_{1}, u_{2}, u_{3}\right)^{T}$, where $T$ denotes transpose operation. Using the polar coordinate $(r=1, \xi, \eta)$, the vector $\vec{u}$ is represented as follows

$$
\vec{u}=(\sin \xi \cos \eta, \sin \xi \sin \eta, \cos \xi)^{T}, \quad 0 \leq \xi \leq \pi, \quad 0 \leq \eta \leq 2 \pi .
$$

We define $\mathrm{u}$ as a vector whose components are monomials of $u_{1}, u_{2}$ and $u_{3}$ with order p:

$$
u \equiv\left(u_{1}^{p}, u_{2}^{p}, u_{3}^{p}, \ldots, u_{1}^{i} u_{2}^{2} u_{3}^{k}, \ldots\right)^{T}, \quad p=i+j+k .
$$

Using moments corresponding to components of the vector $u$, we define a vector $m$ as

$$
m \equiv\left(M_{p 00}, M_{0 p 0}, M_{00 p}, \ldots, \frac{p !}{i ! j ! k !} M_{i j k}, \ldots\right)^{T}
$$

Furthermore, we define $\mathrm{y}$ as a vector whose components are spherical harmonics $Y_{\ell}^{m}$, $m=-\ell,-\ell+1, \ldots, \ell-1, \ell$ :

$$
\mathrm{y}=\left(Y_{p}^{p}, \ldots, Y_{p}^{-p}, Y_{p-2}^{p-2}, \ldots, Y_{p-2}^{-p+2}, \ldots, Y_{\ell}^{m}, \ldots\right)^{T},
$$

where the minimal value of $\ell$ is 0 for even $p$ and is 1 for odd $p$. The monomials $u_{1}^{i} u_{2}^{j} u_{3}^{k}$ in (16) are functions defined on the unit sphere and are represented by linear combinations of spherical harmonics $Y_{\ell}^{m}$ in (18), that is, there is a nonsingular complex matrix A such that

$$
u=A \mathrm{y},
$$

$$
\begin{gathered}
A=\left(A_{i j k, \ell m}\right), i+j+k=p, \quad \ell=p, p-2, \ldots, \ell_{0}, \ell_{0}=0 \text { for even } p,=1 \text { for odd } p, \\
m=-\ell,-\ell+1, \ldots, \ell-1, \ell, \\
A_{i j k, l m}=\int_{0}^{2 \pi} \int_{0}^{\pi}\left[Y_{\ell}^{m}(\xi, \eta)\right]^{*} u_{1}^{i} u_{2}^{j} u_{3}^{k} \sin \xi d \xi d \eta
\end{gathered}
$$

where ${ }^{*}$ denotes a complex conjugate. Then, we define a complex moment vector $\nu$ as $[6]$

$$
\begin{gathered}
\nu \equiv A^{\dagger} m, \\
\nu=\left(\nu_{p}^{p}, \ldots, \nu_{p}^{-p}, \nu_{p-2}^{p-2}, \ldots, \nu_{p-2}^{-p+2}, \ldots, \nu_{t}^{m}, \ldots\right)^{T},
\end{gathered}
$$

where $A^{\dagger}$ is the hermitian conjugate of $A$. It is known [6] that under rotation the vector $\nu$ is transformed according to the same block-diagonal matrix as the vector $\mathbf{y}$. Since spherical harmonics $Y_{1}^{1}, Y_{1}^{0}$ and $Y_{1}^{-1}$ are represented as

$$
Y_{1}^{ \pm 1}=\mp \frac{1}{2} \sqrt{\frac{3}{2} \pi}(x \pm j y), \quad Y_{1}^{0}=\frac{1}{2} \sqrt{\frac{3}{2} \pi} z, j \equiv \sqrt{-1}, \quad x^{2}+y^{2}+z^{2}=1,
$$

we obtain the following relations

$$
x=\sqrt{2 \pi / 3}\left(Y_{1}^{-1}-Y_{1}^{1}\right), \quad y=j \sqrt{2 \pi / 3}\left(Y_{1}^{-1}+Y_{1}^{1}\right), \quad z=2 \sqrt{\pi / 3} Y_{1}^{0} .
$$


Since the vector $(x, y, z)^{T}$ is transformed by rotation matrix $\mathrm{R}$ under rotation, and the complex moment $\nu_{l}^{m}$ is transformed in the same fashion as the spherical harmonics $Y_{\ell}^{m}$ under rotation, the vector $\mathrm{b}=\left(b_{1}, b_{2}, b_{3}\right)^{T}$ defined as

$$
b_{1}=\sqrt{2 \pi / 3}\left(\nu_{1}^{-1}-\nu_{1}^{1}\right), \quad b_{2}=j \sqrt{2 \pi / 3}\left(\nu_{1}^{-1}+\nu_{1}^{1}\right), \quad b_{3}=2 \sqrt{\pi / 3} \nu_{1}^{0},
$$

is transformed by rotation matrix $R$. Let vectors $b$ and $b^{\prime}$ be vectors computed by (25) from density functions of $3-D$ object before and after rotation, respectively. Then, it holds that

$$
\mathbf{b}^{\prime}=R \mathbf{b}
$$

\section{Rotation Inference by Singular Value Decomposition}

Let $b_{1}$ and $b_{2}$ be two column vectors defined as (25) for the 3-D object before rotation, and $a_{1}$ and $a_{2}$ be two similar vectors corresponding to $b_{1}$ and $b_{2}$ for the object after rotation. We define $3 \times 2$ dimensional matrices $A$ and $B$ as

$$
A \equiv\left[\begin{array}{ll}
\mathrm{a}_{1} & \mathrm{a}_{2}
\end{array}\right], \quad B \equiv\left[\begin{array}{ll}
\mathrm{b}_{1} & \mathrm{~b}_{2}
\end{array}\right] .
$$

Let $A B^{T}$ be represented by singular value decomposition as

$$
A B^{T}=U D V^{T}
$$

where

$$
U U^{T}=V V^{T}=I, \quad D=\operatorname{diag}\left(d_{1}, d_{2}, d_{3}\right), \quad d_{1} \geq d_{2} \geq d_{3} \geq 0, \quad I=\text { identity matrix. }
$$

For the case where $\operatorname{rank}\left(A B^{T}\right) \geq 2$, the rotation matrix $\mathrm{R}$ minimizing $\|A-R B\|^{2}$ under the constraints $\operatorname{det}(\mathrm{R})=1$ and $R R^{T}=I$ is given by [7]

$$
R=U S V^{T}
$$

where

$$
S=\operatorname{diag}(1,1, \operatorname{det}(U) \operatorname{det}(V)) \text {. }
$$

\section{3-D Object Positioning Algorithm}

The proposed estimation algorithm for 3-D object positioning from two monocular image brightnesses without point correspondences is summarized as follows. In this section, we use the camera coordinate system $(x, y, z)$.

(Step 1) Estimate $\left(f_{i j}, g_{i j}\right)$ from monocular image brightnesses of object before and after rotation by iterative algorithm (12) and (13). Infer the 3-D shapes $z_{i j}$ of object before and after rotation by integrating $\left(p_{i j}, q_{i j}\right)$ computed from $\left(f_{i j}, g_{i j}\right)$, where $p_{i j}$ and $q_{i j}$ are $p$ and $q$ defined as (3) at the point $(i, j)$ in the image plane, and $z_{i j}$ is the height at an image coordinate $(\mathrm{x}, \mathrm{y})=(\mathrm{i}, \mathrm{j})$. The density function $\rho\left(x^{\prime}, y^{\prime}, z^{\prime}\right)$ in (14) where $x^{\prime}=x-\bar{x}, y^{\prime}=y-\bar{y}, z^{\prime}=z-\bar{z}$ and $(\vec{x}, \vec{y}, \vec{z})$ is the centroid, is given by

$$
\begin{aligned}
\rho\left(x^{\prime}, y^{\prime}, z^{\prime}\right) & =1 & & \text { for } x^{\prime}=i-\bar{x}, y^{\prime}=j-\bar{y}, z^{\prime}=z_{i j}-\bar{z}, \\
& =0 & & \text { otherwise. }
\end{aligned}
$$

(Step 2) For the density function $\rho\left(x^{\prime}, y^{\prime}, z^{\prime}\right)$ in step 1 of object before rotation, compute moments $M_{\ell m n}, \ell+m+n=3$, and $M_{\ell^{\prime} m^{\prime} n^{\prime}}, \ell^{\prime}+m^{\prime}+n^{\prime}=5$ by (14), and construct vectors $\mathrm{b}_{1}$ and $\mathrm{b}_{2}$ corresponding to $M_{l m n}$ and $M_{l^{\prime} m^{\prime} n^{i}}$ by (25), respectively. For $\rho\left(x^{\prime}, y^{\prime}, z^{\prime}\right)$ of object after rotation, compute vectors $\mathbf{a}_{1}$ and $\mathbf{a}_{2}$ similar to $\mathrm{b}_{1}$ and $\mathbf{b}_{2}$ by $(25)$. 
(Step 3) Obtain the rotation matrix $\mathrm{R}$ by (27) through (29).

(Step 4) Estimate rotation axis $\mathrm{e}=\left(e_{1}, e_{2}, e_{3}\right),\|\mathrm{e}\|=1$, and rotation angle $\theta$ around the axis by the following relations.

$$
\begin{gathered}
R \equiv\left(\begin{array}{lll}
r_{1} & r_{2} & r_{3} \\
r_{4} & r_{5} & r_{6} \\
r_{7} & r_{8} & r_{9}
\end{array}\right), \quad d^{2} \equiv\left(r_{8}-r_{6}\right)^{2}+\left(r_{3}-r_{7}\right)^{2}+\left(r_{4}-r_{2}\right)^{2}, \\
\quad \sin \theta= \pm d / 2, \quad \cos \theta=\frac{d^{2} r_{1}-\left(r_{8}-r_{6}\right)^{2}}{d^{2}-\left(r_{8}-r_{6}\right)^{2}} \\
e_{1}= \pm\left(r_{8}-r_{6}\right) / d, \quad e_{2}= \pm\left(r_{3}-r_{7}\right) / d, \quad e_{3}= \pm\left(r_{4}-r_{2}\right) / d,
\end{gathered}
$$

where \pm denotes the sign of same upper or lower side.

\section{Experimental Results}

In order to evaluate the performance of the present 3-D positioning algorithm quantitatively, we show the results of numerical experiments. We use an ellipsoidal object which is represented in the world coordinate system $(X, Y, Z)$ as follows

$$
\frac{X^{2}}{a^{2}}+\frac{Y^{2}}{b^{2}}+\frac{Z^{2}}{c^{2}}=1
$$

We assume that the rotation axis coincides with Z-axis, that the direction of light coincides with the viewing direction perpendicular to the image plane and that the surface material exhibits Lambertian reflection. Examples of the image brightnesses of the ellipsoid of $a=25, b=40$ and $c=25$ before and after the rotation whose angle $\theta$ is $40^{\circ}$, are illustrated in Fig. 1 (a) and (b), respectively, in case of the light direction of $\theta_{i}=90^{\circ}$ and $\phi_{i}=10^{\circ}$, where $\theta_{i}$ and $\phi_{i}$ represent the azimuth and zenith angles of the light direction $n_{i}$ in the world coordinate system $(X, Y, Z)$. Figure 2 shows the corresponding 3-D shapes recovered from the image brightnesses shown in Fig. 1. The estimation errors of rotation angle $\theta$ are shown against view angle $\phi_{i}$ in Fig. 3 as the average over $\theta=10^{\circ}, 20^{\circ}, \ldots, 90^{\circ}$, where $\theta_{i}=90^{\circ}$ and we denote by model 1 the ellipsoid of $a=c=25$ and $b=40$, and by model 2 the ellipsoid of $a=20, b=30$ and $c=25$. It is found that the rotation angle is estimated with the accuracy of error less than few degrees in case of small view angle. In the experiments, at the $\operatorname{step}(2)$ in algorithm in the preceding section, the principal axis of object is used as the vector $\mathrm{b}_{2}$ or $\mathrm{a}_{2}$ instead of the vector constructed from the moment $M_{\ell^{\prime} m^{\prime} n^{\prime}}, \ell^{\prime}+m^{\prime}+n^{\prime}=5$, because the moment of order 5 is too sensitive to noise.

\section{Conclusion}

We have proposed the algorithm for 3-D object positioning from two monocular image brightnesses. The algorithm has the advantageous property that it does not need point correspondence while previous methods need the point correspondence. The numerical experimental results show that by the proposed algorithm, rotation angle is estimated with the accuracy of error less than few degrees in case of small view angle. 


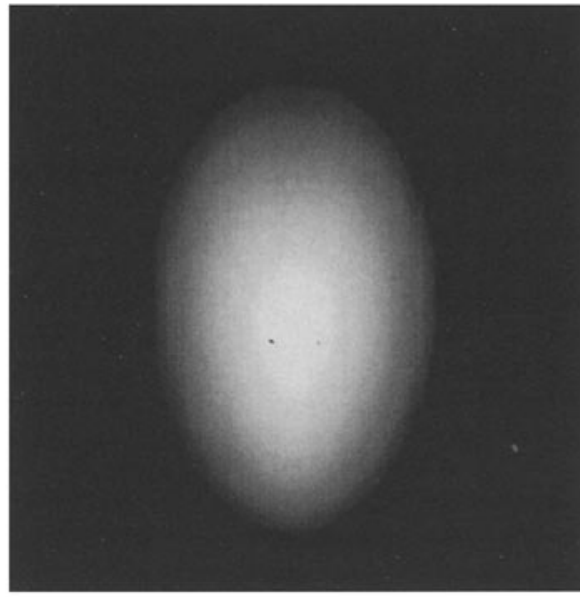

(a)

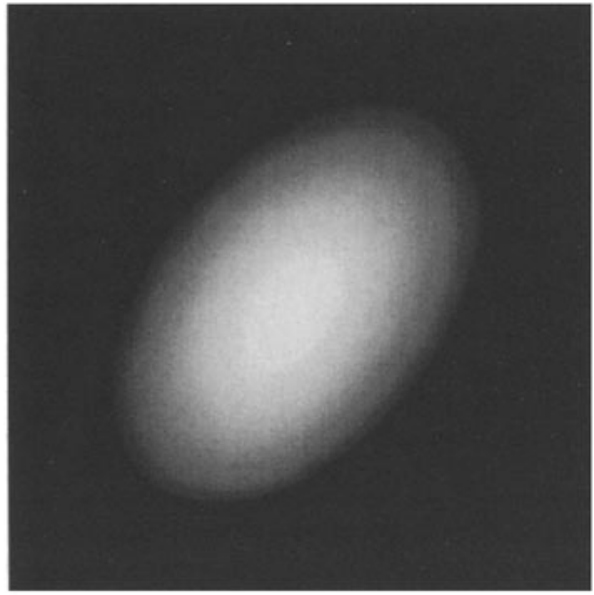

(b)

Fig.1 Image brightnesses of an ellipsoidal object of $a=c=25$ and $b=40$ (a)before and (b) after rotation of angle $\theta=40^{\circ}$, where $\theta_{i}=90^{\circ}, \phi_{i}=10^{\circ}$.

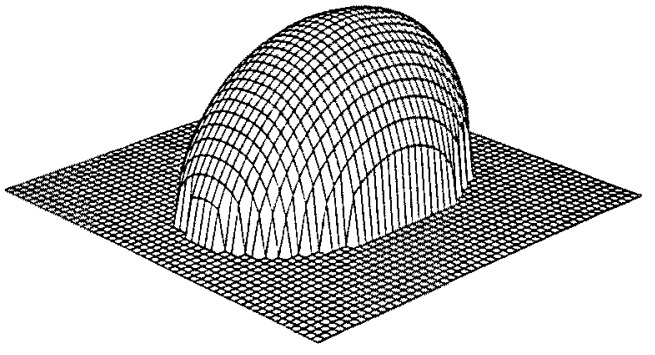

(a)

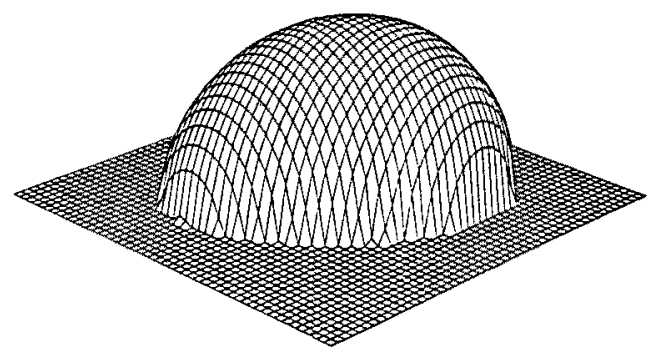

(b)

Fig.2 The corresponding 3-D shapes recovered from the image brightnesses shown in Fig.1.

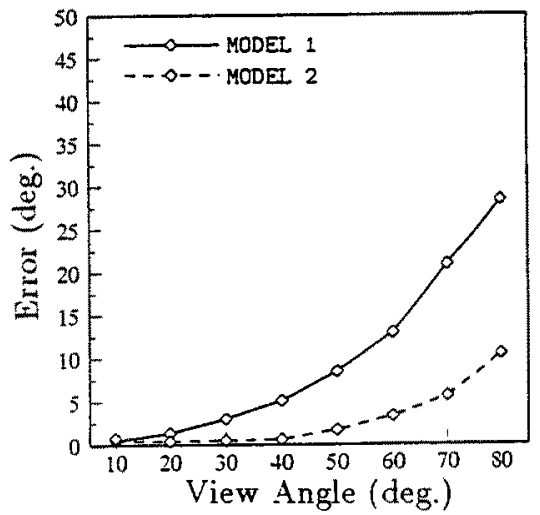

Fig. 3 The estimation errors of rotation angle against view angle $\phi_{i} ;$ we denote by model 1 the ellipsoid of $a=c=25$ and $b=40$, and by model 2 that of $a=20, b=30$ and $c=25$. 


\section{References}

[1]R.Y.Tsai and T.S.Huang,"Uniqueness and Estimation of Three-Dimensional Motion Parameters of Rigid Objects with Curved Surfaces," IEEE Trans. Vol.PAMI-6, No.1 (1984) pp.13-27.

[2]O.D. Faugeras and M.Hebert,"A 3-D Recognition and Positioning Algorithm Using Geometrical Matching between Primitive Surfaces," Proc.8th Int.Joint Conf. Artificial Intell.(1983)pp.996-1002.

[3]J.Weng, T.S.Huang and N.Ahuja,"Error Analysis of Motion Parameter Estimation from Image Sequences, "Proc.1st Int.Conf.Comput.Vision (1987)pp.703-707.

[4]J.Philip,"Estimation of Three-Dimensional Motion of Rigid Objects from Noisy Observations,"IEEE Trans. Vol.PAMI-13,No.1(1991)pp.61-66.

[5]K.Kanatani,"Unbiased Estimation and Statistical Analysis of 3-D rigid Motion from Two Views,"IEEE Trans. Vol.PAMI-15,No.1(1993) pp.37-50.

[6]C.H.Lo and H.S.Don,"3-D Moment Forms:Their Construction and Application to Object Identification and Positioning," IEEE Trans. Vol.PAMI-11,No.10(1989)pp.10531064.

[7]S.Umeyama," Least-Squares Estimation of Transformation Parameters Between Two Point Patterns,"IEEE Trans. Vol.PAMI-13,No.4(1991)pp.376-380. 